\title{
Future Directions for Dementia Risk Reduction and Prevention Research: An International Research Network on Dementia Prevention Consensus
}

Kaarin J. Anstey ${ }^{\mathrm{a}, \mathrm{b}, *}$, Ruth Peters ${ }^{\mathrm{a}, \mathrm{b}}$, Lidan Zheng ${ }^{\mathrm{a}, \mathrm{b}}$, Deborah E. Barnes ${ }^{\mathrm{c}, \mathrm{d}, \mathrm{e}}$, Carol Brayne ${ }^{\mathrm{f}}$, Henry Brodaty $^{\mathrm{g}}$, John Chalmers ${ }^{\mathrm{h}}$, Linda Clare ${ }^{\mathrm{i}}$, Roger A. Dixon ${ }^{\mathrm{j}}$, Hiroko Dodge ${ }^{\mathrm{k}, 1, \mathrm{~m}}$, Nicola T. Lautenschlager ${ }^{\mathrm{n}, \mathrm{o}}$, Laura E. Middleton ${ }^{\mathrm{p}}$, Chengxuan Qiu $^{\mathrm{q}}$, Glenn Rees $^{\mathrm{r}}$, Suzana Shahar and Kristine Yaffe $\mathrm{e}^{\mathrm{c}, \mathrm{d}, \mathrm{e}, \mathrm{t}}$

${ }^{a}$ UNSW Aging Futures Institute, School of Psychology, University of New South Wales, Sydney, NSW, Australia ${ }^{\mathrm{b}}$ Neuroscience Research Australia (NeuRA), Randwick, NSW, Australia

${ }^{\mathrm{c} D e p a r t m e n t}$ of Psychiatry and Behavioral Sciences, University of California, San Francisco, San Francisco, CA, USA

${ }^{\mathrm{d}}$ Department of Epidemiology and Biostatistics, University of California, San Francisco, San Francisco, CA, USA

${ }^{\mathrm{e}}$ San Francisco Veterans Affairs Health Care System, San Francisco, CA, USA

${ }^{\mathrm{f}}$ Cambridge Public Health, University of Cambridge, Forvie Site, Cambridge, UK

${ }^{\mathrm{g}}$ Centre for Healthy Brain Ageing, School of Psychiatry, University of New South Wales, Sydney, NSW, Australia

${ }^{\mathrm{h}}$ Faculty of Medicine, The George Institute for Global Health, University of New South Wales, Sydney, NSW, Australia

${ }^{\mathrm{i}}$ College of Medicine and Health, University of Exeter, St Luke's Campus, Exeter, UK

${ }^{\mathrm{j}}$ Neuroscience and Mental Health Institute, Department of Psychology, University of Alberta, Edmonton, AB, Canada

${ }^{\mathrm{k}}$ Layton Aging and Alzheimer's Disease Center, Department of Neurology, Oregon Health \& Science University, Portland, OR, USA

${ }^{1}$ Michigan Alzheimer's Disease Center, Department of Neurology, University of Michigan, Ann Arbor, MI, USA m Michigan Alzheimer's Disease Center, University of Michigan, Ann Arbor, MI, USA

${ }^{\mathrm{n}}$ Academic Unit for Psychiatry of Old Age, Department of Psychiatry, The University of Melbourne, Parkville, VIC, Australia

${ }^{\circ}$ NorthWestern Mental Health, Royal Melbourne Hospital, Parkville, VIC, Australia

${ }^{\mathrm{p}}$ Department of Kinesiology, University of Waterloo, Waterloo, Ontario, Canada

${ }^{\mathrm{q}}$ Aging Research Center and Center for Alzheimer Research, Department of Neurobiology, Care Sciences and Society, Karolinska Institutet, Solna, Sweden

\footnotetext{
${ }^{*}$ Correspondence to: Kaarin J. Anstey, Neuroscience Research Australia, 139 Barker St, Randwick, NSW 2031, Australia. Tel.: +61 29399 1019; E-mail: k.anstey@unsw.edu.au.
} 


\author{
${ }^{\mathrm{r}}$ Alzheimer's Disease International, London, UK \\ ${ }^{\mathrm{s}}$ Center for Healthy Ageing \& Wellness, Faculty of Health Sciences, Universiti Kebangsaan Malaysia, Jalan \\ Raja Muda Abdul Aziz, Kuala Lumpur, Malaysia \\ ${ }^{\mathrm{t}}$ Department of Neurology, University of California, San Francisco, San Francisco, CA, USA
}

Accepted 30 July 2020

\begin{abstract}
In the past decade a large body of evidence has accumulated on risk factors for dementia, primarily from Europe and North America. Drawing on recent integrative reviews and a consensus workshop, the International Research Network on Dementia Prevention developed a consensus statement on priorities for future research. Significant gaps in geographical location, representativeness, diversity, duration, mechanisms, and research on combinations of risk factors were identified. Future research to inform dementia risk reduction should fill gaps in the evidence base, take a life-course, multi-domain approach, and inform population health approaches that improve the brain-health of whole communities.
\end{abstract}

Keywords: Multi-domain, primary prevention, risk factor, risk reduction

Globally, dementia is one of the top 10 most burdensome health conditions among older people [1]. Although reports of a reduction in incidence in some high-income countries are promising $[2,3]$, prevalence will continue to increase overall due to population aging (e.g., [4]). Furthermore, the impact of rising levels of obesity and diabetes, especially among young people, may counteract declining levels of vascular risk factors (e.g., reduction in smoking rates, levels of blood pressure) over the past two decades [5-7]. In the currently challenging and changing landscape of a world with COVID-19, it is important to optimize overall health of older persons, and produce low-cost, remote health promotion responses to chronic conditions. This will require shifting paradigms for dementia risk reduction. We need to move beyond granular individual risk factor studies and debates about measures and definitions, toward integrating life-course perspectives, person centered outcomes, and policy-level approaches that improve cognition in whole populations.

The International Research Network on Dementia Prevention (IRNDP) [8] brings together researchers and policymakers who are working on dementia prevention via dementia risk reduction, across the globe. The goal is to develop the international evidence base for translating dementia risk reduction research into practice by enhancing information sharing and catalyzing interdisciplinary collaboration. At our first international conference in October 2019, the IRNDP leadership committee held a workshop of experts to develop a position paper on future directions for research on dementia prevention and dementia risk reduction. This built on a special issue focused entirely on dementia prevention and published by the IRNDP in the Journal of Alzheimer's Disease in 2019 alongside multiple key commentaries [9-11]. In this commentary, we present the IRNDP statement on the state of dementia risk reduction and dementia prevention and identify future directions for research that focus primarily on non-pharmacological strategies or pharmacological management of chronic disease (e.g., blood pressure lowering using medication). The aim is to provide clarity for funding bodies, clinicians, research teams and policy makers, and to optimize research efficiency (e.g., [12]).

The reduction of incident dementia cases at a given age is referred to as 'prevention of dementia' at the population level. Because dementia occurs mostly in the very old, delaying the average age of dementia onset by as little as a year or two will lead to a reduction in age specific prevalence as older adults reach life expectancy.

Early work by committee members $[13,14]$ and other recent reports such as the Lancet Commission [15] concluded that childhood education, exercise, maintaining social engagement, reducing smoking, and management of hearing loss, depression, diabetes, and obesity across the life course are key protective factors which collectively have potential to delay or prevent a third of dementia cases. The weight of evidence at present suggests that latelife cognitive decline and dementia are amenable to modification by treatment of vascular risk 


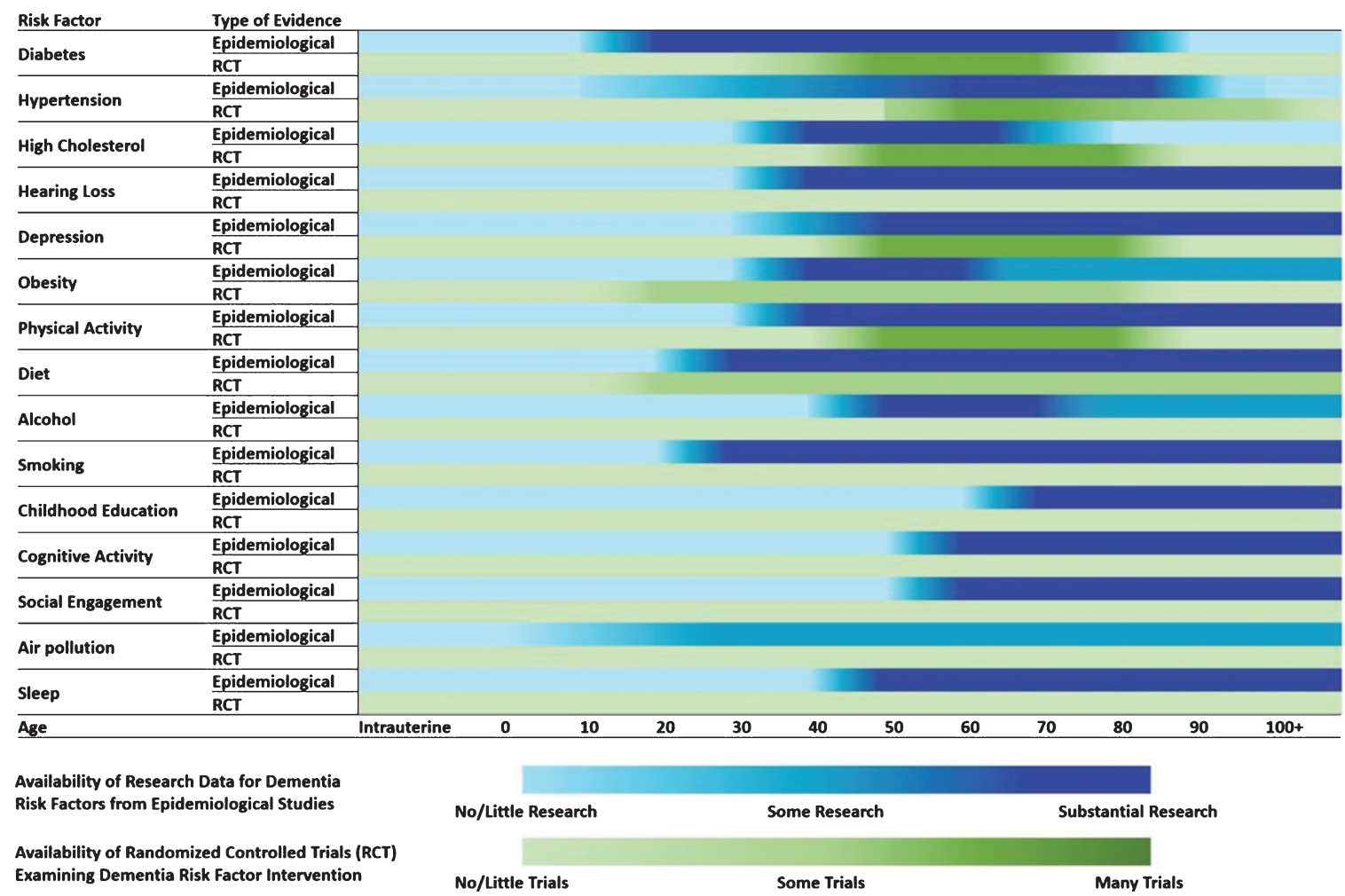

Fig. 1. Schematic Birdseye View of the Current Landscape of Evidence for Dementia Risk Reduction Research. Note. This heatmap is indicative of the evidence that is currently available from systematic reviews and meta-analyses of dementia risk reduction research. Evidence was collated from a number of large-scale reviews [17-20] and influenced by [13-16, 46, 47].

factors, increase in physical, mental, and social activity, and avoiding environmental hazards [16, 17]. The list of modifiable risk factors continues to grow with publication of systematic reviews that allow for aggregation of findings. Examples of newer risk factors that were not included in seminal early papers [13] include sleep disturbance, atrial fibrillation, anxiety, cancer, carotid atherosclerosis, inflammatory markers, metabolic syndrome, peripheral artery disease, renal disease, serum uric acid, stroke, and pesticides [18].

To develop this statement, the IRNDP convened a workshop of experts from several disciplines and six countries. In preparation for the workshop, a high-level summary of evidence gaps was also produced (Fig. 1). To inform this process, members of IRNDP consulted the evidence briefs that underpin the World Health Organization Guidelines on Risk Reduction of Cognitive Decline and Dementia [19], as well as other recent reports that have synthesized evidence from both clinical trials and observational studies (e.g., [15, 16, 20]). Members of IRNDP also performed a systematic review of meta-analyses of all observational studies on risk factors for dementia [18]. This umbrella review conducted an evaluation of the geographical location of source studies for observational evidence, as well as an evaluation of age of exposure, length of followup and consistency of measures from observational studies.

A report was drafted from the workshop and then circulated to the IRNDP Advisory Committee. The report was revised until all authors achieved consensus on the position paper. This report advances the dementia prevention agenda by identifying important gaps in our knowledge and evidence-base on the life-course influences on late-life risk of dementia. It also identifies areas where methodological issues may limit progress, and some considerations for the development of policy for dementia risk reduction and prevention.

The results reported here are the views of the IRNDP regarding the state of dementia risk reduction and prevention research in early 2020 . We first describe several important gaps in knowledge resulting from lack of available data. 
Populations: Ethnic and cultural diversity, geographical location

\section{Gap}

In dementia risk factor research there are significant gaps in the consideration of geographical location and ethnic and cultural diversity, and this has been noted in World Health Organization guidelines [19]. Such knowledge is important for improving population-level and personal preventive programs as well as helping to develop more precise lifestyle and pharmacological interventions and public health messaging across ethnic, cultural and geographical subgroups. Specific areas that need to be addressed are described below:

a. Data gaps in the geographical location from which primary data are available, particularly concerning exposures that are influenced by culture, climate, and country factors. An umbrella review identified that the majority of literature on risk and protective factors for dementia is from Europe and North America, with relatively poorer coverage of data from Oceania, Asia, and South America [18] for many key risk factors. This is particularly concerning for risk factors that are likely to have a strong cultural influence (e.g., diet and leisure activities) or for which there are already different definitions in other chronic disease areas related to ethnicity (e.g., body mass index [21]) and genetic predisposition [22]. Within Europe, there is a lack of epidemiological data from Eastern and Middle European countries [23].

b. Evidence is lacking on specific population groups and cultures within populations. Within countries and cohort studies it is possible that risk exposure, risk effects and protective mechanisms (e.g., cognitive resilience, cognitive reserve, coping mechanisms and strategies) vary within subgroups (e.g., indigenous populations in Australia [24], Canada and United States [25]; racial minorities in the United States (e.g., African Americans, Asian Americans [26]); and neuro-diverse populations (e.g., autism spectrum disorder [27])). These populations are often overlooked due to the need to aggregate data for publication or high-level policy reports.

c. Evidence is lacking on sex and gender effects within and between populations. The field is increasingly aware of the need to evaluate risk profiles stratified by sex and gender to inform sex and gender-based risk reduction strategies [28].

d. Lack of data on contemporary, representative populations. The majority of the cohort studies from which risk factor data have been derived are now out of date, few are truly population representative, and there is little evidence for good comparison across different countries and between sub-groups within populations [29].

\section{Summary and recommendation}

There is an imbalance in availability of data across countries as well as sub-groups within countries. Additionally, there is lack of consideration of between-country and within-country race/ethnic, sex/gender and regional differences in risk exposure. It would be beneficial for the field to improve its understanding of intercultural or intercountry differences as this will provide insights into region-specific risk factor associations and modifications. Understanding regional risk profiles also will help improve and focus local public health initiatives within communities.

\section{Life-course approach: Pinning down timing, duration, and specificity of exposures}

\section{Gap}

There is a lack of understanding of risk exposure and protective effects over the life-course. Much research into dementia focuses on later life cohorts. There is a need to understand how environmental and genetic factors influence the brain and late-life dementia risk from conception to early life, adolescence, young adulthood, and middle age. Specifically, there are gaps in the data on age of exposure and lifecourse stage at which risk and protective factors have been studied. Figure 1 provides a high-level summary of evidence gaps at different stages of the life-course. There is also a lack of information on those aged 85 and over in both cohort studies and clinical trials. In addition, when evidence is synthesized, often age of exposure is not considered and studies with varying baseline ages are pooled. For example, studies that commenced in middle-age may be combined with studies that commenced in late-life. This means that messages about prevention cannot be tailored to specific ages or may even be based on incorrect information.

\section{Summary and recommendation}

We need to move beyond identification of risk factors to characterizing the parameters or patterns of 
exposure over the life-course that are critical. There is a need now to identify the exposure timing and duration at which risk factors become adverse and at which protective factors generate optimal beneficial effects. Ideally by understanding more about the exposure timing and duration, we can specify an optimal timing and dose for interventions for the key risk and protective factors (e.g., physical activity, blood pressure lowering, protective dietary patterns, cognitive and social engagement, and blood glucose). Methodologically, the field could benefit from greater precision in the definition and measurement of exposures from all domains, including using continuous or ordinal scales rather than binary exposure measures (e.g., clinical diagnosis versus no clinical diagnosis) to identify optimal ranges and cut-off points for risk factors and interventions (e.g., hypercholesterolemia, nutrients). Additionally, the creation, validation, and inclusion of measures that are valid across different age-groups are needed so that change can be reliably measured.

\section{Risk and protective factors: Mechanisms and interactions}

Gap

Results from both multi-domain trials and singledomain trials have been inconsistent. This may be due to imperfect understanding of underlying mechanisms leading to sub-optimal trial design, and lack of consideration of interactions between risk factors. With many new trials underway there will be increasing opportunities to understand mechanisms. Similarly, there is a need to identify protection enhancing mechanisms and related interventions to promote cognitive resilience in high-risk individuals and communities. Knowledge gaps exist in the area of mechanisms as follows:

a. Lack of evidence relating to the biological mechanisms underpinning some risk factors raising the issue of whether the risk factors are actually proxies for third variables. For example, there is little understanding currently regarding the biological mechanisms underlying psychosocial factors such as social engagement that appear protective in their presence and risk-elevating in their absence. It is also possible that social engagement is a proxy measure for higher socio-economic status, better sensory function, cognitive activity, or better mental health status (e.g., free from depression).
It is unclear what mechanisms underlie the relationship between adiposity, obesity, and dementia. It is possible that obesity may affect brain and dementia risk indirectly via its association with glycemic control and be a proxy measure or part-proxy measure (i.e., there may be both direct and indirect effects of obesity on brain health and dementia risk, e.g., [5]). High levels of visceral adiposity also may be a marker of subclinical disease (for example, reflecting poorer eating habits and lower physical activity level in someone whose cognitive function is declining), since some studies suggest that body mass index declines approximately ten years prior to dementia diagnosis.

Although multifactorial clinical trials are becoming increasingly popular, single factor clinical trials with biological markers could advance our knowledge of underlying mechanisms of currently broadly defined social engagement and cognition. For example, future randomized controlled clinical trials specifically targeted to increase social interaction and measure concurrent neurobiological changes can help clarify whether there is a causal association between social engagement and cognitive function and can help elucidate underlying mechanisms for the effects (e.g., [30]).

b. There is surprisingly little data published on specific combinations of risk factors. We do not yet understand how reduction in one factor may impact on another; for example, the combination of physical activity and blood pressure lowering. Rigorously designed trials that evaluate interactions between levels of risk factors are needed. Similarly, data from observational studies could be used to evaluate interactions or joint effects of risk factor combinations [31].

c. Another important knowledge gap relating to mechanisms is the understanding of effects of risk factor reversal. For each risk factor, there is a need to find out if reversal of the risk factor also reverses risk of dementia and whether there are thresholds for duration of exposure at which risk reversal does not result in risk reduction of late-life dementia. An example is seen with exercise interventions (for adults with insufficient levels of physical activity) that result in cognitive improvement [32] but such examples need to be extended to establish reduction in dementia incidence in large samples. It would also be possible to follow research approaches in the field of 
smoking where risk reversal has been extensively studied [33].

d. We understand little about the mechanisms underlying cognitive resilience, cognitive reserve and related constructs. While cognitive reserve has long been identified in the literature to explain the impact of factors such as education and enriched environments on brain development, the neurobiological factors underpinning reserve and mechanisms to build reserve in the population, are not understood [34, 35]. Cognitive reserve has been used as a predictor, outcome, and explanatory variable in research. There is a need to distinguish cognitive reserve from other protective lifestyle factors to clarify how cognitive reserve is different from the neuroprotective effects of physical activity and diet. Additional terms that are relevant to this area are 'resistance' and 'compensation' [36]. The field would benefit from consensus regarding conceptual and operational definitions of reserve, resilience and related constructs, and clarification of their neurobiological substrates [37]. As education is a modifiable risk factor affecting whole populations, the potential benefits of promoting cognitive reserve may be enormous.

\section{Summary and recommendation}

We need more understanding of the mechanisms underpinning (and interactions associated with) observed benefits of reducing risk factors and increasing protective factors (e.g., social engagement, education, cognitive resilience and reserve) in order to inform the most efficient and effective multidomain interventions. Methodologically, the first step in achieving this is the specification of levels of risk factors, e.g., rather than 'high education' or 'high levels of physical activity', levels need to be specified in meaningful units of measurement. For some risk factors there needs to be specification of intensity (e.g., physical activity) or dose (e.g. nutrients, cognitive training). This will help us compare strength of association, consistently and specifically across studies as well as pinpoint any dose-response relationships to help establish causal mechanisms.

\section{Interventions: Study design and inconsistent results}

\section{Gap}

Significant progress in dementia prevention research requires optimal design of intervention studies, yet many methodological, measurement and scientific knowledge gaps need to be addressed for this to occur. Key issues include:

a. Lack of long-term follow-up of trials of risk reduction interventions. In part due to the recency of dementia risk reduction trials and the length of time over which neuropathology accumulates, we still lack long-term follow-up of interventions in which onset of dementia is the primary outcome. This will require long-term investment in cohorts that enable assessment of environmental exposures and history effects such as emerging treatments for chronic disease and other health conditions such as COVID-19. Similarly, we need long-term follow-up of randomized controlled trials to allow time to truly evaluate the impact of interventions on incident dementia. Further gains will be achieved by ensuring consistency of outcome measures, inclusion of biomarkers, and optimal clinical characterization. There is potential for big data approaches to accelerate research findings. For example, by enabling analysis of biobanks to test hypotheses, or to apply simulations based on health registries and observational studies. Such advances may increase the rapidity of results and their translation into practical outcomes.

b. Lack of consistency in the findings from observational studies and randomized controlled trials. Another important issue that the field has not yet addressed is the discrepancy in findings between observational studies that identify risk factors and clinical trials testing treatments of those risk factors. For example, statins are associated with reduced risk of dementia in observational studies but have shown no benefit in trials. This phenomenon of 'mismatch' is important to resolve because it has implications for risk modification.

\section{Summary and recommendation}

To fill these gaps, we will need trials that are designed to answer research questions by inclusion of relevant outcome measures, adequate duration of interventions for measurable impact on cognitive function, and adequate length of follow up to demonstrate both efficacy and maintenance of behavioral or policy level change. There is also a need to critically evaluate the appropriateness of comparators in clinical trials and to develop standards for comparators [38]. Capacity building in the areas of big data and data-driven analytics will be critical for progress [39]. This will enable pragmatic and optimal use of 
big datasets (e.g., country level data, administrative data, online data, medical records, genomics, etc.) linked to trial datasets which would enhance longterm follow-ups.

\section{Dementia subtypes: Vascular dementia and rarer neurodegenerative dementias}

Gap

Most of the existing literature on dementia risk factors and risk reduction focuses on all-cause dementia or Alzheimer's disease and ignores other forms of dementia. While basic scientists progress understanding of the pathobiology that causes specific subtypes of dementia, population-level approaches to dementia risk reduction will continue to focus on clinical syndromes. Risk reduction research needs to straddle this tension between a push for increased phenotyping while recognizing that mixed dementia is the most common type of dementia presenting clinically in adults aged over 80 . In particular:

a. There is a limited quantity of research on vascular cognitive impairment and vascular dementia from both observational studies and trials. Most risk reduction trials have focused on cognitive function and all-cause dementia (e.g., [40]) and there is a lack of risk reduction trials that specify dementia driven by vascular pathology as a primary or secondary outcome [41]. Similarly, our systematic review of the observational evidence on risk factors for dementia identified 34 risk factors that have been studied in relation to Alzheimer's disease but only 8 that have been studied in relation to vascular dementia. Knowledge of variation in the rate of progression of sub-types of dementia over the life-course in addition to their specific relationships with risk factors, will inform preventive strategies.

b. Lack of data on risk factors for rarer types of dementia and younger onset dementia. Epidemiological studies rarely have resources to include the assessments required to subtype less prevalent forms of dementia and even where this is possible, small sample sizes often preclude reliable estimates of effect sizes. This means that alternative methods, such as case-control studies, large-scale register-based studies, and data pooling, may be required to obtain better information on risk factors associated with dementias such as frontotemporal [42], Lewy body demen- tia [43], limbic-predominant age-related TDP-43 encephalopathy [44], younger onset dementia [45], etc. While research into the autosomal dominant dementias has focused on pharmaceuticals (e.g., DIAN [46]), there is also a need to determine the extent to which younger onset dementias could be delayed by risk modification, (e.g., [47]).

\section{Summary and recommendation}

Evaluation of risk reduction interventions targeted to specific dementia subtypes, and inclusion of subtyping of dementia as secondary outcomes in large trials, will increase our knowledge about how to reduce risk and prevent dementia due to causes other than Alzheimer's disease.

\section{General summary and recommendations}

Evidence on dementia risk reduction has the potential for enormous impacts on population health. Pre-COVID-19 observational research studies that commenced several decades ago are currently used to inform trial design globally. For example, leading multi-domain clinical trials such as FINGER, PreDIVA, and MYB, as well as other trials that are in development or in progress such as the US POINTER Trial, the MIND-China Trial, the SINGER Trial, CAN-THUMBS UP, HATICE, PRODEMOS, and SMARRT have been developed largely from evidence obtained in cohort studies [48]. Looking forward, research in dementia risk reduction will need to be highly collaborative, long term, take populationlevel perspectives, be interdisciplinary and include outcomes that are meaningful to individuals as well as health practitioners. Multi-domain interventions will need to be evaluated not only for efficacy, but also for cost, participant burden, adherence [49] and practicality. Focus also needs to be given to facilitating efficient and effective knowledge implementation into the community and clinical settings. Ultimately successful community or population level risk reduction interventions will improve the health of whole communities.

There is a general need to recognize that culture and country will influence the risk profile of a population. The population attributable risk of the key risk factors (e.g., insufficient physical activity, midlife hypertension, poor diet) differ between countries and cultures. As the evidence accumulates, we will increasingly be able to develop approaches at three levels: population-level policies and advice, strategies for sub-groups or regions with 
specific vulnerabilities or risks, as well as personalized/individualized risk assessment and intervention. A life-course approach to research will help us to understand long-term causal pathways and determine the optimal timing for different interventions over the life-course.

In addition to developing a research agenda that will address the important questions identified here, scientists need to quantify what success will look like to governments (e.g., compression of morbidity leading to increases in life expectancy free of cognitive impairment and reduction in health care costs; increased cognitive reserve, which will enable older adults to age more productively; and the understanding of mechanisms of disease and risk factors, which will allow for the design of more effective interventions), as well as allocating more resources to educating and training the public, health professionals and policy makers. This will enable us to use the knowledge we currently have to engage governments and policy makers to conduct dementia risk reduction at a higher level.

Specifically, governments need to place more focus on addressing what can be done as a society to reduce dementia risks. For example, optimizing brain development in infancy and childhood as well as providing the necessary resources for ongoing education could help improve cognitive reserve for all citizens. Reducing inequalities is key. The reduction of collective exposures that lead to lifelong blood pressure trajectories will not only abate one of the biggest risk factors for poor brain health but help increase health overall across all age groups. Improving nation-wide physical activity standards as well as areas such as salt intake reduction require multifaceted solutions. These will demonstrate improvements in rates of obesity and diabetes as well as having direct and mediating effects on cognitive health.

The current climate has brought to light the need to improve overall health across the whole population. Older adults and individuals with underlying health conditions have been the most heavily affected by the COVID-19 pandemic. Combined with increasing understanding that risk reduction for dementia needs to be addressed across the life course, this raises a call to action for interventions that can lift the health of whole communities. It is only through collective action that we can hope to implement wide-scale change.

The IRNDP concludes that research in dementia risk reduction is at an exciting juncture. Highly significant research advances have been made with many promising trials underway. We hope our statement will contribute to defining directions, focusing research efforts and facilitating collaboration across research domains and geographic locations.

\section{ACKNOWLEDGMENTS}

The IRNDP is funded by the Australian Government as part of the NHMRC Dementia Collaborative Research Centre (DCRC). KJA is funded by NHMRC Fellowship 1102694. RP and LZ are funded by the DCRC. CQ received grants from the Swedish Research Council (VR, grants no.: 2017-00740 and 2017-05819), the Swedish Foundation for International Cooperation in Research and Higher Education (STINT, grant no. CH2019-8320), and Karolinska Institutet, Stockholm, Sweden.

Authors' disclosures available online (https:// www.j-alz.com/manuscript-disclosures/20-0674r1).

\section{REFERENCES}

[1] Prince MJ (2015) World Alzheimer Report 2015: The Global Impact of Dementia: An analysis of prevalence, incidence, cost and trends, Alzheimer's Disease International, London.

[2] Matthews FE, Stephan BCM, Robinson L, Jagger C, Barnes LE, Arthur A, Brayne C, Comas-Herrera A, Wittenberg R, Dening T, McCracken CFM, Moody C, Parry B, Green E, Barnes R, Warwick J, Gao L, Mattison A, Baldwin C, Harrison S, Woods B, McKeith IG, Ince PG, Wharton SB, Forster G, Cognitive Function and Ageing Studies Collaboration (2016) A two decade dementia incidence comparison from the Cognitive Function and Ageing Studies I and II. Nat Commun 7, 11398.

[3] Satizabal CL, Beiser AS, Chouraki V, Chêne G, Dufouil C, Seshadri S (2016) Incidence of dementia over three decades in the Framingham Heart Study. N Engl J Med 374, 523-532.

[4] Yang L, Jin X, Yan J, Jin Y, Yu W, Wu H, Xu S (2016) Prevalence of dementia, cognitive status and associated risk factors among elderly of Zhejiang province, China in 2014. Age Ageing 45, 708-712.

[5] Taylor C, Tillin T, Chaturvedi N, Dewey M, Ferri CP, Hughes A, Prince M, Richards M, Shah A, Stewart R (2013) Midlife hypertensive status and cognitive function 20 years later: The Southall and Brent revisited study. J Am Geriatr Soc 61, 1489-1498.

[6] Bhupathiraju SN, Hu FB (2016) Epidemiology of obesity and diabetes and their cardiovascular complications. Circ Res 118, 1723-1735.

[7] You W-P, Henneberg M (2016) Type 1 diabetes prevalence increasing globally and regionally: The role of natural selection and life expectancy at birth. BMJ Open Diabetes Res Care 4, e000161.

[8] Anstey KJ, Peters R, Clare L, Lautenschlager NT, Dodge HH, Barnes DE, Shahar S, Brodaty H, Rees G (2017) Joining forces to prevent dementia: The International Research Network on Dementia Prevention (IRNDP). Int Psychogeriatr 29, $1757-1760$. 
[9] Glymour MM, Whitmer RA (2019) Using cross-cultural studies to improve evidence on dementia prevention: Lessons from the special issue sponsored by the International Research Network on Dementia Prevention (IRNDP). J Alzheimers Dis 70, S5-S10.

[10] Brayne C, Richard E (2019) Prevention of cognitive decline: A goal in sight? J Alzheimers Dis 70, S11-S14.

[11] Sexton CE, Yaffe K (2019) Population-based approaches to dementia prevention. J Alzheimers Dis 70, S15-S17.

[12] Chalmers I, Glasziou P (2016) Systematic reviews and research waste. Lancet 387, 122-123.

[13] Barnes DE, Yaffe K (2011) The projected effect of risk factor reduction on Alzheimer's disease prevalence. Lancet Neurol 10, 819-828.

[14] Norton S, Matthews FE, Barnes DE, Yaffe K, Brayne C (2014) Potential for primary prevention of Alzheimer's disease: An analysis of population-based data. Lancet Neurol 13, 788-794

[15] Livingston G, Sommerlad A, Orgeta V, Costafreda SG, Huntley J, Ames D, Ballard C, Banerjee S, Burns A, CohenMansfield J, Cooper C, Fox N, Gitlin LN, Howard R, Kales HC, Larson EB, Ritchie K, Rockwood K, Sampson EL, Samus Q, Schneider LS, Selbaek G, Teri L, Mukadam N (2017) Dementia prevention, intervention, and care. Lancet 390, 2673-2734.

[16] Prince M, Albanese E, Guerchet M, Prina M (2014) World Alzheimer's Report 2014. Dementia and Risk Reduction: An analysis of protective and modifiable factors, Alzheimer's Disease International, London.

[17] Kane RL, Butler M, Fink HA, Brasure M, Davila H, Desai P, Jutkowitz E, McCreedy E, Nelson VA, McCarten JR (2017) Interventions to prevent age-related cognitive decline, mild cognitive impairment, and clinical Alzheimer's-type dementia. AHRQ Comp Eff Rev 17, 17-EHC008-EF.

[18] Anstey KJ, Ee N, Eramudugolla R, Jagger C, Peters R (2019) A systematic review of meta-analyses that evaluate risk factors for dementia to evaluate the quantity, quality, and global representativeness of evidence. J Alzheimers Dis 70, S165-S186.

[19] World Health Organization (2019) Risk Reduction of Cognitive Decline and Dementia: WHO guidelines, World Health Organization, Geneva.

[20] National Academies of Sciences, Engineering, and Medicine (2017) Preventing Cognitive Decline and Dementia: A Way Forward, The National Academies Press, Washington, DC.

[21] Davis J, Juarez D, Hodges K (2013) Relationship of ethnicity and body mass index with the development of hypertension and hyperlipidemia. Ethn Dis 23, 65-70.

[22] Licher S, Ahmad S, Karamujić-Čomić H, Voortman T, Leening MJ, Ikram MA, Ikram MK (2019) Genetic predisposition, modifiable-risk-factor profile and long-term dementia risk in the general population. Nat Med 25, 13641369.

[23] Kiejna A, Frydecka D, Adamowski T, Bickel H, Reynish E, Prince M, Caracciolo B, Fratiglioni L, Georges J (2011) Epidemiological studies of cognitive impairment and dementia across Eastern and Middle European countries (epidemiology of dementia in Eastern and Middle European Countries). Int J Geriatr Psychiatry 26, 111-117.

[24] Radford K, Lavrencic LM, Delbaere K, Draper B, Cumming R, Daylight G, Mack HA, Chalkley S, Bennett H, Garvey G, Hill TY, Lasschuit D, Broe GA (2019) Factors associated with the high prevalence of dementia in older Aboriginal Australians. J Alzheimers Dis 70, S75-S85.
[25] MacDonald JP, Barnes DE, Middleton LE (2015) Implications of risk factors for Alzheimer's disease in Canada's Indigenous population. Can Geriatr J 18, 152.

[26] Babulal GM, Quiroz YT, Albensi BC, Arenaza-Urquijo E, Astell AJ, Babiloni C, Bahar-Fuchs A, Bell J, Bowman GL, Brickman AM (2019) Perspectives on ethnic and racial disparities in Alzheimer's disease and related dementias: Update and areas of immediate need. Alzheimers Dement 15, 292-312.

[27] Roestorf A, Bowler DM, Deserno MK, Howlin P, Klinger L, McConachie H, Parr JR, Powell P, Van Heijst BFC, Geurts HM (2019) "Older Adults with ASD: The Consequences of Aging." Insights from a series of special interest group meetings held at the International Society for Autism Research 2016-2017. Res Autism Spectr Disord 63, 3-12.

[28] Ferretti MT, Iulita MF, Cavedo E, Chiesa PA, Dimech AS, Chadha AS, Baracchi F, Girouard H, Misoch S, Giacobini E (2018) Sex differences in Alzheimer disease-the gateway to precision medicine. Nat Rev Neurol 14, 457-469.

[29] Pickett J, Bird C, Ballard C, Banerjee S, Brayne C, Cowan K, Clare L, Comas-Herrera A, Corner L, Daley S, Knapp M, Lafortune L, Livingston G, Manthorpe J, Marchant N, Moriarty J, Robinson L, van Lynden C, Windle G, Woods B, Gray K, Walton C (2018) A roadmap to advance dementia research in prevention, diagnosis, intervention, and care by 2025. Int J Geriatr Psychiatry 33, 900-906.

[30] Dodge HH, Zhu J, Mattek NC, Bowman M, Ybarra O, Wild KV, Loewenstein DA, Kaye JA (2015) Web-enabled conversational interactions as a method to improve cognitive functions: Results of a 6-week randomized controlled trial. Alzheimers Dement (N Y) 1, 1-12.

[31] Sapkota S, Huan T, Tran T, Zheng J, Camicioli R, Li L, Dixon RA (2018) Alzheimer's biomarkers from multiple modalities selectively discriminate clinical status: Relative importance of salivary metabolomics panels, genetic, lifestyle, cognitive, functional health and demographic risk markers. Front Aging Neurosci 10, 296.

[32] Lautenschlager NT, Cox KL, Flicker L, Foster JK, van Bockxmeer FM, Xiao J, Greenop KR, Almeida OP (2008) Effect of physical activity on cognitive function in older adults at risk for Alzheimer disease: A randomized trial. JAMA 300, 1027-1037.

[33] Dresler CM, Leon ME, Straif K, Baan R, Secretan B (2006) Reversal of risk upon quitting smoking. Lancet 368, 348-349.

[34] Jones RN, Manly J, Glymour MM, Rentz DM, Jefferson AL, Stern Y (2011) Conceptual and measurement challenges in research on cognitive reserve. J Int Neuropsychol Soc 17, 593-601.

[35] Stern Y, Zarahn E, Hilton HJ, Flynn J, DeLaPaz R, Rakitin B (2003) Exploring the neural basis of cognitive reserve. $J$ Clin Exp Neuropsychol 25, 691-701.

[36] Montine TJ, Cholerton BA, Corrada MM, Edland SD, Flanagan ME, Hemmy LS, Kawas CH, White LR (2019) Concepts for brain aging: Resistance, resilience, reserve, and compensation. Alzheimers Res Ther 11, 22.

[37] Stern Y, Barnes CA, Grady C, Jones RN, Raz N (2019) Brain reserve, cognitive reserve, compensation, and maintenance: Operationalization, validity, and mechanisms of cognitive resilience. Neurobiol Aging 83, 124-129.

[38] Freedland KE, King AC, Ambrosius WT, Mayo-Wilson E, Mohr DC, Czajkowski SM, Thabane L, Collins LM, Rebok GW, Treweek SP, Cook TD, Edinger JD, Stoney CM, Campo RA, Young-Hyman D, Riley WT, National Institutes of Health Office of Behavioral and Social Sciences Research 
Expert Panel on Comparator Selection in Behavioral and Social Science Clinical Trials (2019) The selection of comparators for randomized controlled trials of health-related behavioral interventions: Ecommendations of an NIH expert panel. J Clin Epidemiol 110, 74-81.

[39] Badhwar A, McFall GP, Sapkota S, Black SE, Chertkow H, Duchesne S, Masellis M, Li L, Dixon R, Bellec P (2019) A multiomics approach to heterogeneity in Alzheimer's disease: Focused review and roadmap. Brain 143, 13151331.

[40] SPRINT MIND Investigators for the SPRINT Research Group, Williamson JD, Pajewski NM, Auchus AP, Bryan RN, Chelune G, Cheung AK, Cleveland ML, Coker LH, Crowe MG, Cushman WC, Cutler JA, Davatzikos C, Desiderio L, Erus G, Fine LJ, Gaussoin SA, Harris D, Hsieh MK, Johnson KC, Kimmel PL, Tamura MK, Launer LJ, Lerner AJ, Lewis CE, Martindale-Adams J, Moy CS, Nasrallah IM, Nichols LO, Oparil S, Ogrocki PK, Rahman M, Rapp SR, Reboussin DM, Rocco MV, Sachs BC, Sink KM, Still CH, Supiano MA, Snyder JK, Wadley VG, Walker J, Weiner DE, Whelton PK, Wilson VM, Woolard N, Wright JT, Jr., Wright CB (2019) Effect of intensive vs standard blood pressure control on probable dementia: A randomized clinical trial. JAMA 321, 553-561.

[41] Gustafson DR (2018) Epidemiology informs randomized clinical trials of cognitive impairments and late-onset, sporadic dementias. J Neurol Neuromed 3, 13-18.

[42] Onyike CU, Diehl-Schmid J (2013) The epidemiology of frontotemporal dementia. Int Rev Psychiatry 25, 130-137.

[43] Javanshiri K, Haglund M, Englund E (2019) Cardiovascular disease, diabetes mellitus, and hypertension in Lewy body disease: A comparison with other dementia disorders. J Alzheimers Dis 71, 851-859.
[44] Nelson PT, Dickson DW, Trojanowski JQ, Jack CR, Boyle PA, Arfanakis K, Rademakers R, Alafuzoff I, Attems J, Brayne C (2019) Limbic-predominant age-related TDP-43 encephalopathy (LATE): Consensus working group report. Brain 142, 1503-1527.

[45] Nordström P, Nordström A, Eriksson M, Wahlund L-O, Gustafson Y (2013) Risk factors in late adolescence for young-onset dementia in men: A nationwide cohort study. JAMA Intern Med 173, 1612-1618.

[46] Bateman RJ, Benzinger TL, Berry S, Clifford DB, Duggan C, Fagan AM, Fanning K, Farlow MR, Hassenstab J, McDade EM (2017) The DIAN-TU Next Generation Alzheimer's prevention trial: Adaptive design and disease progression model. Alzheimers Dement 13, 8-19.

[47] Casaletto K, Staffaroni A, Wolf A, Appleby B, Brushaber D, Coppola G, Dickerson B, Domoto-Reilly K, Elahi F, Fields J (2020) Active lifestyles moderate clinical outcomes in autosomal dominant frontotemporal degeneration. Alzheimers Dement 16, 91-105.

[48] Bott NT, Hall A, Madero EN, Glenn JM, Fuseya N, Gills JL, Gray M (2019) Face-to-face and digital multidomain lifestyle interventions to enhance cognitive reserve and reduce risk of Alzheimer's disease and related dementias: A review of completed and prospective studies. Nutrients 11, 2258.

[49] Turunen M, Hokkanen L, Backman L, Stigsdotter-Neely A, Hanninen T, Paajanen T, Soininen H, Kivipelto M, Ngandu T (2019) Computer-based cognitive training for older adults: Determinants of adherence. PLoS One 14, e0219541. 


\section{University Library}

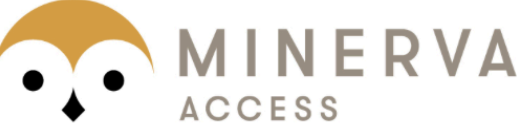

A gateway to Melbourne's research publications

Minerva Access is the Institutional Repository of The University of Melbourne

\section{Author/s:}

Anstey, KJ;Peters, R;Zheng, L;Barnes, DE;Brayne, C;Brodaty, H;Chalmers, J;Clare, L;Dixon, RA;Dodge, H;Lautenschlager, NT;Middleton, LE;Qiu, C;Rees, G;Shahar, S;Yaffe, K

Title:

Future Directions for Dementia Risk Reduction and Prevention Research: An International Research Network on Dementia Prevention Consensus

Date:

2020-01-01

\section{Citation:}

Anstey, K. J., Peters, R., Zheng, L., Barnes, D. E., Brayne, C., Brodaty, H., Chalmers, J., Clare, L., Dixon, R. A., Dodge, H., Lautenschlager, N. T., Middleton, L. E., Qiu, C., Rees, G., Shahar, S. \& Yaffe, K. (2020). Future Directions for Dementia Risk Reduction and Prevention Research: An International Research Network on Dementia Prevention Consensus. JOURNAL OF ALZHEIMERS DISEASE, 78 (1), pp.3-12. https://doi.org/10.3233/ JAD-200674.

Persistent Link:

http://hdl.handle.net/11343/271933

License:

CC BY-NC 